\title{
ON WIMAN BOUND FOR ARITHMETIC RIEMANN SURFACES
}

\author{
MIKHAIL BELOLIPETSKY \\ Sobolev Institute of Mathematics, Koptyuga 4, 630090 Novosibirsk, Russia, Max Planck Institute of \\ Mathematics, Vivatsgasse 7, 53111 Bonn, Germany \\ e-mail:mbel@math.nsc.ru \\ and GRZEGORZ GROMADZKI \\ Institute of Mathematics University of Gdańsk, Wita Stwosza 57, 80-952 Gdańsk, Poland \\ e-mail: greggrom@math.univ.gda.pl
}

(Received 22 February 2002; accepted 12 April, 2002)

\begin{abstract}
We show that the order of an automorphism of an arithmetic Riemann surface of genus $g$ is not greater than $2 g-2$, provided $g$ is large enough. This bound is an arithmetic analog of the classical Wiman bound. We prove that it is sharp and attained for any genus but in contrast to the general case the automorphisms of maximal order act without fixed points. This allows us to consider the automorphisms which act on arithmetic Riemann surfaces and have a given number of fixed points. For these automorphisms we describe the asymptotic behavior of their orders.
\end{abstract}

2000 Mathematics Subject Classification. 30F10, 14H45.

1. Introduction. This paper continues a project on exploration of the connections between arithmetic and geometric properties of hyperbolic manifolds, especially of hyperbolic Riemann surfaces, started in [1], [2]. In the first paper the upper bound for the order of the group of automorphisms of a non-arithmetic Riemann surface was obtained. This gives a non-arithmetic analog of the classical Hurwitz theorem. The result of the second paper is an arithmetic analog of Accola-Maclachlan lower bound for the maximal order of the group of automorphisms of a surface. Now we focus our attention on the maximal order of a single conformal automorphism acting on a Riemann surface.

In the classical setting there is a well known result of Wiman [14] that any automorphism of a Riemann surface of genus $g \geq 2$ has order not greater then $4 g+2$ and this bound is attained for any $g$. The automorphism of the maximal order has always 1 fixed point. In [11] it was shown that any automorphism with 2 fixed points has order not greater than $4 g$, and in [6] that any automorphism with $k>2$ fixed points has order not greater than $2 g /(k-2)+1$. By [5] the later bounds are also attained for arbitrary $g$ and $k$ for which $2 g /(k-2)$ is an integer.

We restrict ourselves to the arithmetic Riemann surfaces; that is, the surfaces uniformized by arithmetic Fuchsian groups. In Section 2 we show that for $g \geq 11$, $g \neq 15$, the order of any automorphism of an arithmetic Riemann surface of genus $g$ is not greater then $2 g-2$, this bound is also sharp and attained for any genus. In contrast to the general case corresponding automorphisms always act without fixed points unless their order is 2 . This does not mean that automorphisms of arithmetic 
Riemann surfaces of order greater then 2 act without fixed points, of course. However, in Section 3 we prove that for the automorphisms which act on arithmetic surfaces and have $k \geq 1$ fixed points $\lim _{g \rightarrow \infty} n(g, k) / g=0$, where $n(g, k)$ denotes the maximal order of an automorphism acting on a surface of genus $g$ with $k$ fixed points.

For the sake of economy we do not devote separate section for preliminaries. The principal tool is Borel's discreteness theorem for the covolumes of arithmetic groups [3]. Our methods are based on the uniformisation theorem, the theory of Fuchsian groups and known classification results for 2-generator arithmetic Fuchsian groups. Actually, all the required facts and definitions can be found in [2]. We can also recommend [4] as a source of information concerning methodology of combinatorial study of Riemann surfaces and their groups of automorphisms.

2. Arithmetic Analog of Wiman Bound. Here we shall study the bound for the order of an automorphism of an arithmetic Riemann surface of genus $g \geq 2$. The first theorem shows that the general bound of Wiman fails to be sharp in the case of such surfaces.

THEOREM 2.1. Given $g \geq 2$ there is an arithmetic Riemann surface of genus $g$ admitting a group of automorphism of order $2(g-1)$. Conversely, if $g$ is large enough then any automorphism of an arithmetic surface of genus $g$ has order not exceeding $2(g-1)$.

Proof. We first construct an arithmetic Riemann surface of genus $g \geq 2$ with an automorphism of order $n=2(g-1)$. Let $\Delta$ be an arithmetic group with signature $(1 ; 2,2)$; eg a subgroup of index 2 of an arithmetic Fuchsian group with signature $(1 ; 2)$ which exists by [13]. Consider an epimorphism $\theta: \Delta=\left\langle a, b, x_{1}, x_{2}\right| x_{1}^{2}, x_{2}^{2}$, $\left.[a, b] x_{1} x_{2}\right\rangle \rightarrow \mathbf{Z}_{n}=\langle x\rangle$ defined by $\theta(a)=\theta(b)=x$ and $\theta\left(x_{1}\right)=\theta\left(x_{2}\right)=x^{g-1}$. Then it is clearly a surface-kernel epimorphism, which shows that $x$ represents an automorphism of order $2(g-1)$ acting on an arithmetic Riemann surface of genus $g$ in virtue of the Riemann-Hurwitz formula. This proves the first part of the theorem.

Now assume that an automorphism $\varphi$ of an arithmetic Riemann surface $X$ has order $n$. Then $\langle\varphi\rangle \cong \Delta / \Gamma$, where $\Delta$ is an arithmetic Fuchsian group say with signature $\left(\gamma ; m_{1}, \ldots, m_{r}\right)$ and $\Gamma=\Gamma_{g}$ is a Fuchsian surface group uniformizing $X$. By Riemann-Hurwitz formula $n=\mu(\Gamma) / \mu(\Delta)=4 \pi(g-1) / \mu(\Delta)$. There is nothing to do if $\mu(\Delta) \geq 2 \pi$ since in this case $n \leq 2(g-1)$. So assume that $\mu(\Delta)<2 \pi$. We shall show that $\gamma>0$ if $g$ is sufficiently large.

Consider set $\Sigma_{2 \pi}=\left\{\sigma_{i}\right\}_{i \in I}$ of all signatures with area smaller than $2 \pi$ and with orbit genus 0 which can appear as signatures of arithmetic Fuchsian groups. By Borel's discreteness theorem [3] $I$ is finite. Let $\mu=\operatorname{Max}\left\{\mu\left(\sigma_{i}\right) \mid i \in I\right\}$. Now if $\Delta_{i}$ is a Fuchsian group with signature $\left(0 ; m_{1}, \ldots, m_{r}\right)$ from $\Sigma_{2 \pi}$ and $\theta_{i}: \Delta_{i} \rightarrow \mathrm{Z}_{N_{i}}$ is an epimorphism then $N_{i} \leq M_{i}=1$. c.m. $\left\{m_{1}, \ldots, m_{r}\right\}$. Let $N=\operatorname{Max}\left\{M_{i} \mid i \in I\right\}$ and define $g_{0}=[\mu N / 4 \pi]+1$, where [] denotes the integral part.

Coming back to our situation, if $\gamma=0$ then $4 \pi(g-1)=\mu(\Delta) n \leq \mu N$ which means that $g \leq g_{0}$. So if $g>g_{0}$ then $\gamma>0$ indeed. But if $\gamma>1$ then $\mu(\Delta) \leq 4 \pi$ which gives $n \leq g-1$. So we are interested in $\gamma=1$. Let $a, b, x_{1}, \ldots, x_{r}$ be a set of canonical generators for $\Delta$ and let $\theta: \Delta \rightarrow \mathrm{Z}_{n} \cong\langle\varphi\rangle$ be surface-kernel epimorphism. Then $\theta\left(a b a^{-1} b^{-1}\right)=1$ and therefore $r \geq 2$. The minimum possible area in this case is $2 \pi$ that is achieved only for signature $(1 ; 2,2)$, which means $n \leq 2(g-1)$. This completes the proof. 
In a contrast to the general case, the arithmetic Riemann surfaces of sufficiently large genus with automorphisms of maximal order are not unique. Indeed, from [13] follows that there are non-commensurable arithmetic groups of signature $(1 ; 2,2)$. So for any genus $g \geq 2$ there exist non-isometric arithmetic surfaces with automorphisms of order $2(g-1)$. Now let us study the gap sequence in Theorem 2.1.

THEOREM 2.2. The values $g \leq 11$ and $g=15$ are the only ones that can stand as the genera of arithmetic Riemann surfaces admitting automorphisms of order greater then $2(g-1)$.

Proof. To prove this theorem we shall find the signatures that form the set $\Sigma_{2 \pi}$ defined in the proof of Theorem 2.1. By Riemann-Hurwitz formula such signatures are of the form $\left(0 ; m_{1}, \ldots, m_{r}\right)$ with $r=3,4,5$ (for short we shall write $\left(m_{1}, \ldots, m_{r}\right)$ ). To refine $\Sigma_{2 \pi}$ we shall use known classification results about arithmetic Fuchsian groups and the criterion of Harvey [7] by which a surface-kernel epimorphism from such group onto a cyclic group of order $n$ exists if and only if $n=1$. c.m. $\left\{m_{1}, \ldots, m_{r}\right\}=$ 1.c.m. $\left\{m_{1}, \ldots, \hat{m}_{i}, \ldots, m_{r}\right\}$ for any $i$ in range $1 \leq i \leq r$.

Let us start with $r=3$. The list of all the arithmetic triangle groups was obtained by Takeuchi in [12]. By Harvey criterion the only arithmetic triangle groups which admit surface-kernel epimorphisms onto cyclic groups have signatures $(2,5,10),(2,7$, 14), (2, 8, 8), (2, 9, 18), (2, 12, 12), (2, 15, 30), (3, 4, 12), (3, 6, 6), (3, 8, 24), (3, 10, 30), $(3,12,12),(4,8,8),(4,16,16),(5,5,5),(5,10,10),(6,12,12),(6,24,24),(7,7,7),(9$, $9,9),(9,18,18)$ and $(15,15,15)$. They give actions of automorphisms of orders 10, 14 , $8,18,12,30,12,6,24,30,12,8,16,5,10,12,24,7,9,18,15$ on surfaces of genera 2 , $3,2,4,3,7,3,2,7,9,4,3,6,2,4,5,10,3,4,8,7$, respectively.

The helpful observation to enumerate signatures $\left(m_{1}, m_{2}, m_{3}, m_{4}\right)$ from $\Sigma_{2 \pi}$ is that the signatures are determined by the Fuchsian groups up to an ordering of periods, i.e. we can assume that $m_{1} \leq m_{2} \leq m_{3} \leq m_{4}$. Using the mentioned above criterion of Harvey we find that the quadrangle signatures with $\mu<2 \pi$ that admit surface-kernel epimorphisms onto cyclic groups are: $(2,2, m, m)$ with $m \geq 3,(2,3,3,6),(2,3,4,12)$, $(2,3,5,30),(3,3,3,3),(3,3,4,4),(3,3,5,5)$. Let us check which of these signatures can be realized by arithmetic Fuchsian groups. One can see that any group of signature $(2,2, m, m)$ is contained as index 2 subgroup in a $(2,2,2, m)$-group. The usual way to construct normal subgroups of index $n$ is to define epimorphisms from a given group onto finite groups of order $n$. In the cases we need the corresponding epimorphisms can be easily obtained and we shall not write them down. All $(2,2,2, m)$ signatures that admit arithmetic groups are known [9], they correspond to $m=3,4,5,6,7,8$, $9,10,12,14,18,22$. These gives automorphisms of orders $6,4,10,6,14,8,18,10$, $12,14,18$ and 22 acting respectively on surfaces of genera 2, 2, 4, 3, 6, 4, 8, 5, 6, 7, 9 and 11 . Arithmetic groups of signature $(2,3,3,6)$ and $(3,3,4,4)$ can be obtained as subgroups of index 2 in arithmetic triangle groups with signatures $(3,4,12)$ and $(3,8$, $8)$, respectively, where the later groups exist by [12]. An arithmetic group with signature $(3,3,3,3)$ can be choosen as a normal subgroup of index 4 in an arithmetic Fuchsian group with signature $(2,2,2,3)$ existing by [9]. For the remaining three signatures we shall apply Singerman's method [10] which gives necessary and sufficient conditions for a group $\Delta$ to have a non necesarily normal subgroup $\Delta_{1}$ of index $N$ in terms of epimorphisms from $\Delta$ onto finite permutation groups transitive on $N$ points. For signature $(2,3,4,12)$ there exists a group that is contained as a subgroup of index 5 in an arithmetic triangle group with signature $(2,4,12)$ existing by [12]. The corresponding homomorphism $\theta$ from $\left\langle x_{1}, x_{2}, x_{3} \mid x_{1}^{2}, x_{2}^{4}, x_{3}^{12}, x_{1} x_{2} x_{3}\right\rangle$ onto transitive subgroup 
of symmetric group $S_{5}$ is defined by $\theta\left(x_{1}\right)=(1,2)(3,4)(5), \theta\left(x_{2}\right)=(1,5,4,2)(3)$, $\theta\left(x_{3}\right)=(1)(2,3,4,5)$. By [12] there exists an arithmetic group with signature $(2,3$, $30)$, it contains a group with signature $(2,3,5,30)$ as a subgroup of index 7 . The corresponding epimorphism $\theta$ from $\left\langle x_{1}, x_{2}, x_{3} \mid x_{1}^{2}, x_{2}^{3}, x_{3}^{30}, x_{1} x_{2} x_{3}\right\rangle$ onto transitive subgroup of $S_{7}$ is defined by $\theta\left(x_{1}\right)=(1,2)(3,4)(5,6)(7), \theta\left(x_{2}\right)=(7,6,4)(2,1,3)(5)$, $\theta\left(x_{3}\right)=(3,2,4,5,6,7)(1)$. Finally, by [12] there exists an arithmetic Fuchsian group with signature $(3,3,5)$ and it contains a subgroup with signature $(3,3,5,5)$ defined by means of epimorphism $\theta\left(x_{1}\right)=(1,2,3)(4,5,6)(7), \theta\left(x_{2}\right)=(1,3,4)(7,6,5)(2)$, $\theta\left(x_{3}\right)=(1,6,7,4,2)(3)(5)$. Concluding, we obtain automorphisms of orders $6,12,30$, $3,12,15$ acting on surfaces of genera $3,6,15,2,6,8$, respectively.

Finally, by Harvey criterion there are no any signatures with $r=5$ and $\mu<2 \pi$ that admit surface-kernel epimorphisms onto cyclic groups.

From the proof of Theorem 2.2 it follows:

COROLlARY 2.3. All cyclic automorphisms groups of orders exceeding $2(g-1)$ which can act on arithmetic Riemann surfaces of genus $g$ are given in the following table

\begin{tabular}{|c|c|}
\hline Genus & Cyclic groups of automorphisms \\
\hline 2 & $\mathrm{Z}_{10}, \mathrm{Z}_{8}, \mathrm{Z}_{6}, \mathrm{Z}_{5}, \mathrm{Z}_{4}, \mathrm{Z}_{3}$ \\
3 & $\mathrm{Z}_{14}, \mathrm{Z}_{12}, \mathrm{Z}_{8}, \mathrm{Z}_{7}, \mathrm{Z}_{6}$ \\
4 & $\mathrm{Z}_{18}, \mathrm{Z}_{12}, \mathrm{Z}_{10}, \mathrm{Z}_{9}, \mathrm{Z}_{8}$ \\
5 & $\mathrm{Z}_{12}, \mathrm{Z}_{10}$ \\
6 & $\mathrm{Z}_{16}, \mathrm{Z}_{14}, \mathrm{Z}_{12}$ \\
7 & $\mathrm{Z}_{30}, \mathrm{Z}_{24}, \mathrm{Z}_{15}, \mathrm{Z}_{14}$ \\
8 & $\mathrm{Z}_{18}, \mathrm{Z}_{15}$ \\
9 & $\mathrm{Z}_{30}, \mathrm{Z}_{18}$ \\
10 & $\mathrm{Z}_{24}$ \\
11 & $\mathrm{Z}_{22}$ \\
15 & $\mathrm{Z}_{30}$ \\
\hline
\end{tabular}

Observe that the above Corollary combined with Theorem 2.1 solves the minimum genus problem for automorphisms of even order of arithmetic Riemann surfaces.

3. Automorphisms with Fixed Points. A theorem of Macbeath [8] implies that the automorphisms of arithmetic Riemann surfaces of genus $g>11$ and $g \neq 15$ of maximum possible order act without fixed points unless their order is 2 . This does not mean that automorphisms of arithmetic Riemann surfaces of order greater then 2 act without fixed points, of course, but it seems to be rather difficult either to find the general formulae for the maximal order $n(g, k)$ of an automorphism acting on an arithmetic Riemann surface of genus $g$ with $k$ fixed points or even to obtain essential bounds for $n(g, k)$. Instead of, we discovered the following asymptotic behavior of the orders of such automorphisms:

THEOREM 3.1. Let $n(g, k)$ denote the maximum order of an automorphism acting on an arithmetic Riemann surface of genus $g$ with $k$ fixed points. Then

$$
\lim _{g \rightarrow \infty} \frac{n(g, k)}{g}= \begin{cases}2, & \text { for } k=0 \\ 0, & \text { for } k \geq 1\end{cases}
$$


Proof. The formula for $k=0$ follows immediately from Theorem 2.1. So let $k \geq 1$. Clearly, $\lim _{g \rightarrow \infty} n(g, k) / g=\lim _{g \rightarrow \infty} n(g, k) /(g-1)$. Assume to a contrary that there are $k>0$ and $N_{0}$ such that $(g-1) / n(g, k) \leq N_{0}$ for infinitely many values of $g$, say $\left\{g_{i}\right\}_{i \in N}$. Let $\varphi_{i}$ be an automorphism of order $n\left(g_{i}, k\right)$ which acts with $k$ fixed points on an arithmetic Riemann surface of genus $g_{i}$. Then $\langle\varphi\rangle \cong \Delta_{i} / \Gamma$, where $n\left(g_{i}, k\right)=4 \pi\left(g_{i}-1\right) / \mu\left(\Delta_{i}\right)$ and so $\mu\left(\Delta_{i}\right)=4 \pi\left(g_{i}-1\right) / n\left(g_{i}, k\right) \leq 4 N_{0} \pi$. Since by [8] the fixed points of $\varphi_{i}$ are in bijective correspondence with the orders of canonical generators of $\Delta_{i}$ which are equal to the order of $\varphi_{i}$, arbitrary such $\Delta_{i}$ has signature $\left(\gamma ; n\left(g_{i}, k\right), . k . n\left(g_{i}, k\right), m_{1}, \ldots, m_{r}\right)$ for some $\gamma \geq 0$ and $m_{1}, \ldots, m_{r}<n\left(g_{i}, k\right)$.

Let $\mathcal{A}_{k, N_{0}}$ denotes the set of signatures $\left(\gamma ; n, \ldots, n, m_{1}, \ldots m_{r}\right)$ which admit arithmetic groups, have periods $m_{1}, \ldots, m_{r}<n$ and area not exceeding $4 N_{0} \pi$. Then $\mathcal{A}_{k, N_{0}}$ is finite by Borel's discreteness theorem [3]. Let $n_{0}$ be the maximal period that appears among the periods of signatures from $\mathcal{A}_{k, N_{0}}$. Then $n\left(g_{i}, k\right) \geq n_{0}$ and so for $g_{i}>n_{0} N_{0}+1,\left(g_{i}-1\right) / n\left(g_{i}, k\right)>n_{0} N_{0} / n_{0}=N_{0}$ which is a contradiction.

Acknowledgment. The authors are thankful to Caroline Series and Mathematics Research Center of the University of Warwick, where the paper was started out during the workshop on Kleinian Groups and Hyperbolic 3-manifolds.

\section{REFERENCES}

1. M. Belolipetsky, On the number of automorphisms of a nonarithmetic Riemann surface, Sib. Math. J. 38, No. 5 (1997), 860-867.

2. M. Belolipetsky and G. A. Jones, A bound for the number of automorphisms of an arithmetic Riemann surface, Math. Proc. Camb. Phil. Soc., to appear.

3. A. Borel, Commensurability classes and volumes of hyperbolic 3-manifolds, Ann. Scuola Norm. Sup. Pisa Cl. Sci. (4) 8 (1981), 1-33.

4. E. Bujalance, J. J. Etayo Gordejuela, J. M. Gamboa, and G. Gromadzki, Automorphism groups of compact bordered Klein surfaces: A Combinatorial Approach, Lecture Notes in Mathematics, No. 1439 (Springer Verlag, 1990).

5. C. Corrales, J. M. Gamboa and G. Gromadzki, Automorphisms of Klein surfaces with fixed points, Glasgow Math. J. 41 (1999), 183-189.

6. H. M. Farkas and I. Kra, Riemann surfaces, Graduate Text in Mathematics, No. 71 (Springer Verlag, 1980).

7. W. Harvey, Cyclic groups of automorphisms of a compact Riemann surface, Quart. J. Math. Oxford (2) 17 (1966), 86-97.

8. A. M. Macbeath, Action of automorphisms of a compact Riemann surface on the first homology, Bull. London Math. Soc. 5 (1973), 103-118.

9. C. Maclachlan and G. Rosenberger, Two-generator arithmetic Fuchsian groups, II, Math. Proc. Camb. Phil. Soc. 111, No. 7 (1992), 7-24.

10. D. Singerman, Subgroups of Fuchsian groups and finite permutation groups, Bull. London Math. Soc. 2 (1970), 319-323.

11. T. Szemberg, Automorphism of Riemann surfaces with two fixed points, Ann. Polon. Math. 55 (1991), 343-347.

12. K. Takeuchi, Arithmetic triangle groups, J. Math. Soc. Japan 29 (1977), 91-106.

13. K. Takeuchi, Arithmetic Fuchsian groups of signature $(1 ;$ e), J. Math. Soc. Japan 35 (1983), 381-407.

14. A. Wiman, Über die hyperelliptischen Kurven und diejenigen vom Geschlecht $p=3$, welche eindeutige Transformationen in sich zulassen, Bihang Till. Kongl. Svenska VetenskapsAkademiens Handlingar 21, 1, No. 1 (1895), 23 pp. 